\title{
Ichthyofauna of lotic environments in the Ivinhema river basin, upper Paraná river, Mato Grosso do Sul state, Brazil
}

\author{
Wagner Vicentin $^{1,3 *}$, Fabiane Silva Ferreira ${ }^{2}$ \& \& Yzel Rondon Súarez $z^{2}([)$ \\ ${ }^{1}$ Programa de Pós-Graduação em Ecologia e Conservação, Universidade Federal de Mato Grosso do Sul, \\ 79070-900, Campo Grande, MS, Brasil \\ ${ }^{2}$ Laboratório de Ecologia, Centro de Estudos em Recursos Naturais, Universidade Estadual de Mato Grosso do \\ Sul, Rod. Dourados-Itahum, km 12, 79804-970, Dourados, MS, Brasil \\ ${ }^{3}$ Universidade Federal da Grande Dourados, Rua João Rosa Góes, 1761, Vila Progresso, 79825-070, \\ Dourados, MS, Brasil \\ *Corresponding author: Wagner Vicentin, e-mail: wagnervicentin.bio@gmail.com
}

VICENTIN, W., FERREIRA, F. S., SÚAREZ, Y. R. Ichthyofauna of lotic environments in the Ivinhema river basin, upper Paraná river, Mato Grosso do Sul state, Brazil. Biota Neotropica. 19(4): e20190735. http://dx.doi.org/10.1590/1676-0611-BN-2019-0735

\begin{abstract}
In order to inventory the species richness and fish fauna composition along the Ivinhema river basin, 232 stretches of rivers and streams were sampled from 2000 to 2018, using a rectangular sieve, trawls, gillnets, cast nets, and electrofishing. A total of 141 species was caught in the basin, including seven orders and 35 families. For the recorded species, 42 are from the upper Paraná river, 51 from lower Paraná river, 27 from other basins, two from other continents, and the origin for 19 species are unknown/not reported. Six species are registred for the first time in the upper Paraná river basin: Astyanax abramis, Moenkhausia oligolepis, Serrapinnus kriegi, Curimatopsis myersi, Pyxiloricaria menezesi, and Cichlasoma dimerus. This is the first complete survey of the ichthyofauna in lotic environments along the whole Ivinhema river basin, containing relevant information for comparing studies and serving as reference data that can contribute to the management and implementation of politics for conservation of the basin. This survey also increases 24 species on the list of the most complete inventory in the upper Paraná river published in 2007.
\end{abstract}

Keywords: Fish communities, freshwater, survey.

\section{Ictiofauna de ambientes lóticos na bacia do rio Ivinhema, alto rio Paraná, Mato Grosso do Sul, Brasil}

\begin{abstract}
Resumo: Com o objetivo de inventariar a riqueza de espécies e a composição da ictiofauna ao longo da bacia do rio Ivinhema, 232 trechos de rios e riachos foram amostrados de 2000 a 2018, utilizando uma peneira retangular, rede de arrasto, rede de espera, tarrafas e pesca elétrica. Ao todo 141 espécies foram amostradas na bacia, incluindo oito ordens e 35 famílias. Para as espécies registradas, 42 são do alto rio Paraná, 51 do baixo rio Paraná, 27 de outras bacias, duas de outros continentes e para 19 espécies as origens são desconhecidas ou não reportadas. Seis espécies são registradas pela primeira vez na bacia alto rio Paraná: Astyanax abramis, Moenkhausia oligolepis, Serrapinnus kriegi, Curimatopsis myersi, Pyxiloricaria menezesi e Cichlasoma dimerus. Este é o primeiro levantamento da ictiofauna em ambientes lóticos para toda a bacia do rio Ivinhema, contendo informação relevante para estudos comparativos e servindo como dados de referências que podem contribuir para gerenciamento e implementação de políticas para conservação da bacia. Este levantamento também acrescenta 24 espécies na lista do inventário mais completo realizado no alto rio Paraná publicado em 2007.
\end{abstract}

Palavras-chave: Comunidades de peixes, água doce, inventário. 


\section{Introduction}

The upper Paraná river basin, with 367 fish species, being 317 described and 50 not formally described (Langeani et al. 2007), is located entirely within the territory of Brazil and extends from the municipalities of Guaira and Mundo Novo, upstream to the ancient waterfall known as Sete Quedas, which is currently submerged by the Itaipu Reservoir, and encompasses $891,000 \mathrm{~km}^{2}$ or $10.5 \%$ of the area of Brazil (Agostinho et al. 2003). This portion of the Paraná river basin is the most studied regarding Brazilian freshwater fish (Agostinho et al. 2007), but for some regions the knowledge is still insufficient. Most of those studies were performed in the Brazilian Southeast (Paraná and São Paulo states) and despite of increasing researches, there have been only a few studies in the Midwest Brazilian (region where upper Paraná is inserted, including Mato Grosso do Sul and Goiás states and Federal District) (Aquino et al. 2009).

In the particular case of the Ivinhema river basin, a tributary pratically free of impoundments and located in the western portion of upper Paraná river basin, some studies have been conducted to determine the environmental features that influence fish species in some few headwater streams (Súarez et al. 2007, Súarez \& Lima-Junior, 2009), that influence spacial and temporal variation of fish in streams of lower Ivinhema (Súarez 2008a) or the fish richness in streams of the whole Ivinhema basin (Súarez et al. 2011). Fish eggs and larvae distribution related to environmental factors were also studied in the upper Ivinhema and its river tributaries (Nascimento \& Nakatani 2006) and fishfauna associated to macrophyte mats were described in the lower Ivinhema (Bulla et al. 2011). In the latter, in Súarez (2008b) and in Súarez \& Lima-Junior (2009) a small list of 28, 46 and 64 fish species is presented, respectively, and restricted to the lower Ivinhema river. The richness of 111 fish species was reported in Súarez et al. (2011), but no list of fish species was presented. In the current study, more streams and rivers stretches were sampled and then we described a larger fish richness and composition along the whole Ivinhema river basin, including streams and the main river tributaries.

Given that the upper Paraná river basin is located in a densely populated area that is also one of the most industrialized regions in Brazil (Thomaz et al. 2004), it is not surprising that this area has experienced continuous environmental alteration, that may have a substantial influence on fish communities and their habitats (Bryce et al. 1999, Holt et al. 2006). Therefore, studies describing fish community composition and occurrence in the western part of the upper Paraná river basin are needed, where lotic environments, free from impoundment, still exist. This is particularly important in the state of Mato Grosso do Sul, which has experienced over the last decade great expansion of sugar-cane cultivation and alcohol production (Domingues \& Thomaz Junior 2012). Studies, like the present work, would be very important in obtain information useful in the minimization and mitigation of the impacts of anthropic alteration and facilitate management policies for conservation, specifically for the fish fauna.

It is in this context that the present study was conducted, using data from field sampling, with the aim of inventorying the richness and composition of fish species in the Ivinhema river basin.

\section{Material and Methods}

The Ivinhema river is the main tributary of the Paraná river in the western portion of the state of Mato Grosso do Sul. The mouth of this river is in the stretch of the Paraná river below Engenheiro Sérgio Motta dam but above Itaipu hydroelectric dam, which is the only stretch of the Paraná free of impoundments (Agostinho et al. 2000), including the Ivinhema river basin. The headwater is the Dourados river (area $=9,900 \mathrm{~km}^{2}$ ) at $700 \mathrm{~m}$ of altitude, with the contribution of other subbasins including Santa Maria river (area $=2,700 \mathrm{~km}^{2}$ ), Brilhante river $\left(\right.$ area $\left.=8,878 \mathrm{~km}^{2}\right)$, Vacaria river $\left(\right.$ area $\left.=6,500 \mathrm{~km}^{2}\right)$, and the Ivinhema river $\left(18,045 \mathrm{~km}^{2}\right)$, forming the Ivinhema river basin, with a total area of $46,023 \mathrm{~km}^{2}$. Samplings were performed along 232 stretches of rivers and streams, throughout the Ivinhema river basin from January 2000 to June 2018, being that seven stretches were sampled in Súarez (2008b), seven in Súarez \& Lima-Junior (2009), 186 in Súarez (2011) and 32 in the current study (Figure 1).

Fish were sampled from an approximately $100 \mathrm{~m}$ extension of each stream: 40 throws of a $1.2 \times 0.8 \mathrm{~m}$ ( $2 \mathrm{~mm}$ mesh size) rectangular sieve; 10 trawlings (1.5 x $5 \mathrm{~m} ; 2 \mathrm{~mm}$ mesh size); 12 hours of exposition to a sequence of 14 gillnets $(10 \times 1.5 \mathrm{~m}$, with mesh sizes between adjacent knots: 1.5 , 2.0,3.0 4.0, 5.0, 6.0, 7.0, 8.0, 9.0,10.0 cm) and three cast nets (height 1.5 to $3 \mathrm{~m}$, diameter 3 to $6 \mathrm{~m}$, and mesh sizes between adjacent knots: 1.5 , $3.0,6.0 \mathrm{~cm}$ ). Also, electrofishing was employed in small streams, with approximately $100-\mathrm{m}$-long hauls. In rivers, fish were also captured with a series of 14 gillnets and cast nets in each stretch, whereas along the banks of the rivers the rectangular sieve and trawls were used mainly to sample small sized fish.

Fish were anesthetized with Eugenol solution, euthanized and, in the sequence, fixed in $10 \%$ formalin and preserved in $70 \%$ ethanol. The euthanasia followed all the ethic criterition established by the Universidade Estadual de Mato Grosso do Sul, along the accomplishment of all the projects that originated the current data. The collections of specimens were authorized by the Brazilian Environment Institute (IBAMA), through special license number \# 13458-1159001, issued by SISBIO. Taxonomic identifications followed Graça \& Pavanelli (2007) and current taxonomic reviews. Some specimens were deposited in zoological collections: Núcleo de Pesquisas em Limnologia, Ictiologia e Aquicultura da Universidade Estadual de Maringá (NUP/UEM), Museu de Zoologia da Universidade de São Paulo (MZUSP), Museu da Universidade Federal do Rio Grande do Sul (UFRGS), Museu de Zoologia da Universidade Estadual de Londrina (MZUEL) and Coleção de Peixes da Universidade estadual de Mato Grosso do Sul (CPUEMS).

For those species that we were not able to present voucher specimens, we inserted the information of other references that had already reported the ocurrence of the species in the Ivinhema river basin or upper Paraná river basin.

After the list was compiled, in order to confirm species names, possible synonyms and their basin of occurrence, we confronted it with information available on 'Check List of the Freshwater Fishes of South and Central America' (Reis et al. 2003), 'Catálago das Espécies de Peixes de Água Doce do Brasil' (Buckup et al. 2007), 'Catalog of Fishes' (Eschmeyer et al. 2018) and 'Fishbase' (Froese \& Pauly 2018). 


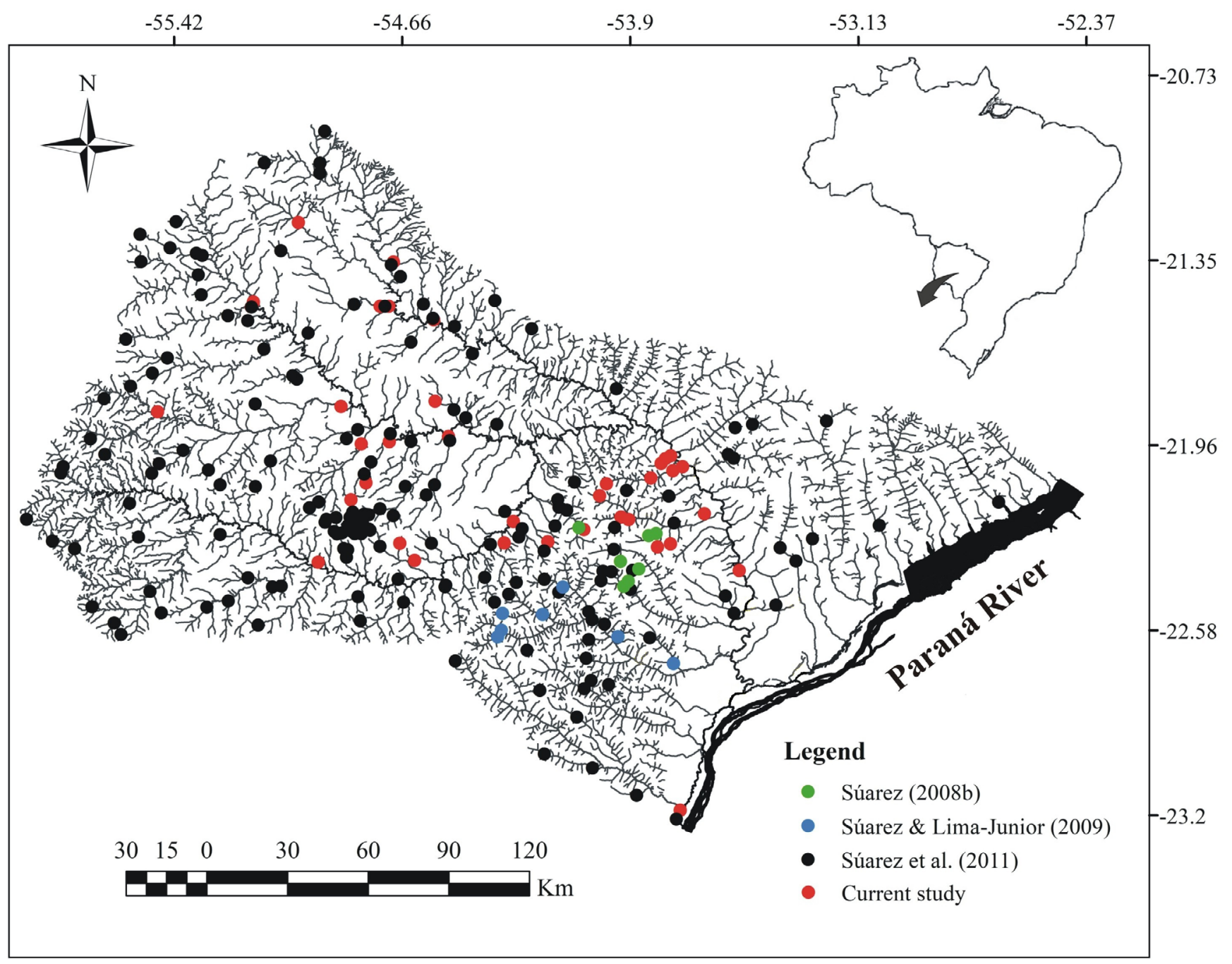

Figure 1. Sampled stretches of rivers and streams that comprise the Ivinhema river basin, in the upper Paraná river basin (between January 2000 and June 2018), Mato Grosso do Sul State, Brazil.

The geographic origin for fish species followed Langeani et al (2007) as autochthonous (native), natural species from the upper Paraná; allochthonous, species from other Neotropical regions and introduced in the upper Paraná; and exotic, species from other continents. For some species not reported in Langeani et al. (2007), the origin was defined according to reviews for the species or the remarks of Ota et al. (2018).

The fish fauna composition based in size was analyzed through the maximum size of each species (standard length for fish that has caudal fin, total length for fish that has only this information available and for Gymnotiformes or Synbranchiformes, and the total disc length for stingrays). This information were gathered from FishBase or directly measured on the specimen in the case of species is not described, not inserted in the database or the sampled specimen was longer than the reported in the FishBase. The size classes were obtained by Sturges formula $(\mathrm{K}=1+3.32 \log \mathrm{n}$, where $\mathrm{K}=$ number of classes; and, $\mathrm{n}=$ the size sample), which furnishes the number of classes for the total sampling, based on the range of total fish size divided by the number of classes.

\section{Results}

A total of 141 species was caught in this study. From this, eight species are without voucher numbers, but we inserted references that already reported these species in the Ivinhema river basin, and only one species we did not find any reference reporting its occurrence in the basin (Table 1). All species are distributed in 7 orders and 35 families. Characiformes and Siluriformes were the most representative orders with $65(46.1 \%)$ and 50 species $(35.5 \%)$, respectively, and Characidae and Loricariidae were the most abundant families with 28 (19.8\%) and 17 species (12\%), respectively. Althought four species were identified at genus level, they were considered different species from those that are already described and reported for the upper Paraná basin.

After verification for synonymies and species that were redescribed, our study also contributed to increase the fish inventory furnished by Langeani et al. (2007), since 24 species were not reported on that list, and six of these species are the first reporting (species with asterisk) in the upper Paraná river: Phalloceros harpagos, 
Vicentin, W. et al.

Table 1. Fish species caught between January 2000 and June 2018 in the Ivinhema river basin and its sub-basins (Santa Maria river - S; Dourados river - D; Vacaria river - V; Brilhante river - B; Ivinhema river - I), upper Paraná river basin. Voucher numbers, size (standard length, total length*, or total disc length** in centimeters) and species origin.

\begin{tabular}{|c|c|c|c|c|}
\hline Species & Voucher or Reference & Size (cm) & sub-basin & origin \\
\hline \multicolumn{5}{|l|}{ MYLIOBATIFORMES } \\
\hline \multicolumn{5}{|l|}{ Potamotrygonidae } \\
\hline Potamotrygon falkneri Castex \& Maciel, 1963 & $\begin{array}{l}\text { Agostinho et al. } 1997, \\
\text { Lonardoni et al. } 2006, \\
\text { Lacerda et al. } 2008\end{array}$ & $78^{* *}$ & $\mathrm{~B}, \mathrm{I}$ & allochthonous \\
\hline Potamotrygon motoro (Müller \& Henle, 1841) & $\begin{array}{l}\text { Agostinho et al. } 1997, \\
\text { Lonardoni et al. } 2006, \\
\text { Lacerda et al. } 2008\end{array}$ & $50 * *$ & $\mathrm{~B}$ & allochthonous \\
\hline
\end{tabular}

\section{CHARACIFORMES}

Acestrorhynchidae

Acestrorhynchus lacustris (Lütken, 1875)

Anostomidae

Leporellus vittatus (Valenciennes, 1850)

Leporinus amblyrhynchus Garavello \& Britiski, 1987

Leporinus friderici (Bloch, 1794)

Leporinus lacustris Campos, 1945

Leporinus octofasciatus Steindachner, 1915

Leporinus striatus Kner, 1859

Megaleporinus macrocephalus (Garavello \& Britski, 1988)*

Megaleporinus piavussu Britski, Birindelli \& Garavello, 2012*

Megaleporinus obtusidens (Valenciennes, 1837)*

Schizodon borellii (Boulenger, 1900)

Bryconidae

Salminus brasiliensis (Cuvier, 1816)

Salminus hilarii Valenciennes, 1850

Characidae

Aphyocharax anisitsi Eigenmann \& Kennedy, 1903

Aphyocharax dentatus Eigenmann \& Kennedy 1903

Aphyocharax sp.

Astyanax abramis (Jenyns, 1842)

Astyanax biotae Castro \&Vari, 2004

Astyanax bockmanni Vari \& Castro, 2007

Astyanax fasciatus (Cuvier ,1819)

Astyanax lacustris (Lütken, 1875)*

Astyanax paranae Eigenmann, 1914

Galeocharax knerii (Steindachner, 1879)

Hyphessobrycon anisitsi (Eigenmann, 1907)

Hyphessobrycon eques (Steindachner ,1882)

Knodus moenkhausii (Eigenmann \& Kennedy, 1903)

Moenkhausia bonita Benine, Castro \& Sabino, 2004

Moenkhausia forestii Benine, Mariguela \& Oliveira, 2009

Moenkhausia intermedia Eigenmann, 1908

Moenkhausia oligolepis (Günther, 1864) Glass tetra

Moenkhausia sanctaefilomenae (Steindachner, 1907)
CPUEMS118

27

S,D,V,B,I autochthonous*

\#

CPUEMS18

NUP9375

NUP9387

CPUEMS424

CPUEMS290

CPUEMS754

NUP9371

CPUEMS301

CPUEMS705

CPUEMS707

NUP9374

CPUEMS261

NUP9944

NUP9361

MZUEL14444

CPUEMS537

Súarez 2008b

CPUEMS116

NUP9378

CPUEMS523

CPUEMS280

CPUEMS170

NUP9314

CPUEMS522

NUP9317

NUP9318

CPUEMS232

CPUEMS531

NUP9936

$\begin{array}{ccc}30 & \text { S,V } & \text { autochthonous } \\ 27.4 & \text { I } & \text { autochthonous } \\ 40 & \text { S,D,V,B,I } & \text { autochthonous* } \\ 20.3 & \text { D,B,I } & \text { autochthonous } \\ 31.2 & \text { S,I } & \text { autochthonous* } \\ 25 & \text { S,D,V,B } & \text { autochthonous } \\ 60 & \text { S,V,B,I } & \text { allochthonous } \\ 40 & \text { D,I } & \text { autochthonous* } \\ 39.8 & \text { I } & \text { autochthonous* } \\ 30 & \text { S,D,V,I } & \text { autochthonous* }\end{array}$

100

50

S,D,V,B,

autochthonous*

S,D, V,B,I

autochthonous*

5.5

7.2

5.3

14

5.2

6.7

12

10.6

11.3

33.1

6

I

$\mathrm{S}, \mathrm{V}, \mathrm{I}$

allochthonous

S,D,V,B,

autochthonous*

I

I

---

S,D,I

S,D,V,B,I

S,D, V,B,I

$\mathrm{D}, \mathrm{V}, \mathrm{B}, \mathrm{I}$

$\mathrm{D}, \mathrm{V}, \mathrm{B}, \mathrm{I}$

D,B,I

S,D,I

4

4.8

4.4

---

D,I

S,D,V,B,I

S,D,B,I

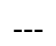

$\mathrm{S}, \mathrm{D}, \mathrm{V}, \mathrm{B}, \mathrm{I}$ unknown

autochthonous

autochthonous

autochthonous*

autochthonous

autochthonous

autochthonous

autochthonous*

allochthonous

unknown

autochthonous*

autochthonous*

unknown

autochthonous* autochthonous*

autochthonous* 
Continuation Table 1.

\begin{tabular}{|c|c|c|c|c|}
\hline Species & Voucher or Reference & Size (cm) & sub-basin & origin \\
\hline Oligosarcus pintoi Campos, 1945 & NUP9315 & 8.4 & $\mathrm{~S}, \mathrm{D}, \mathrm{V}, \mathrm{B}, \mathrm{I}$ & autochthonous \\
\hline Piabarchus stramineus Eigenmann, 1908* & CPUEMS119 & 5.6 & $\mathrm{~S}, \mathrm{D}, \mathrm{V}, \mathrm{B}, \mathrm{I}$ & autochthonous \\
\hline Psellogrammus kennedyi (Eigenmann, 1903) & NUP9321 & 5.9 & $\mathrm{~S}, \mathrm{D}, \mathrm{V}, \mathrm{B}, \mathrm{I}$ & autochthonous* \\
\hline Roeboides descalvadensis Fowler, 1932 & NUP9363 & 8.9 & $\mathrm{~S}, \mathrm{D}, \mathrm{V}, \mathrm{B}, \mathrm{I}$ & allochthonous \\
\hline Serrapinnus kriegi (Schindler, 1937) & MZUEL14348 & 2.4 & I & unknown \\
\hline Serrapinnus notomelas (Eigenmann, 1915) & NUP9937 & 3.6 & $\mathrm{~S}, \mathrm{D}, \mathrm{V}, \mathrm{B}, \mathrm{I}$ & autochthonous \\
\hline Serrapinnus sp. & CPUEMS16 & 3.2 & $\mathrm{D}, \mathrm{V}, \mathrm{B}, \mathrm{I}$ & unknown \\
\hline \multicolumn{5}{|l|}{ Crenuchidae } \\
\hline Characidium zebra Eigenmann, 1909 & NUP9353 & 7.4 & $\mathrm{~S}, \mathrm{D}, \mathrm{V}, \mathrm{B}, \mathrm{I}$ & autochthonous* \\
\hline \multicolumn{5}{|l|}{ Curimatidae } \\
\hline Curimatopsis myersi Vari, 1982 & MZUEL14516 & 4.4 & I & unknown \\
\hline Cyphocharax modestus (Fernández-Yépez, 1948) & NUP9327 & 16.2 & $\mathrm{~S}, \mathrm{D}, \mathrm{V}, \mathrm{B}, \mathrm{I}$ & autochthonous \\
\hline Cyphocharax vanderi (Britski, 1980) & MZUEL14496 & 6.8 & I & autochthonous \\
\hline Steindachnerina brevipinna (Eigenmann \& Eigenmann, 1889) & NUP9365 & 10.9 & $\mathrm{~S}, \mathrm{D}, \mathrm{V}, \mathrm{B}, \mathrm{I}$ & allochthonous \\
\hline Steindachnerina insculpta (Fernández-Yépez, 1948) & NUP9358 & 10.6 & $\mathrm{D}, \mathrm{V}, \mathrm{B}, \mathrm{I}$ & autochthonous \\
\hline \multicolumn{5}{|l|}{ Cynodontidae } \\
\hline Rhaphiodon vulpinus Spix \& Agassiz, 1829 & $\begin{array}{l}\text { Agostinho et al. 1997, } \\
\text { Reynalte-Tataje et al. } \\
2011\end{array}$ & 80 & $\mathrm{~B}, \mathrm{I}$ & autochthonous* \\
\hline \multicolumn{5}{|l|}{ Hemiodontidae } \\
\hline Hemiodus orthonops Eigenmann \& Kennedy, 1903 & CPUEMS275 & 25 & S,I & allochthonous \\
\hline \multicolumn{5}{|l|}{ Lebiasinidae } \\
\hline Pyrrhulina australis Eigenmann \& Kennedy, 1903 & NUP9356 & 5 & $\mathrm{~S}, \mathrm{D}, \mathrm{V}, \mathrm{B}, \mathrm{I}$ & autochthonous* \\
\hline \multicolumn{5}{|l|}{ Parodontidae } \\
\hline Apareiodon affinis (Steindachner, 1879) & Agostinho et al. 1997 & 17 & $\mathrm{D}$ & autochthonous* \\
\hline Apareiodon ibitiensis Campos, 1944 & MZUSP85418 & 11.3 & $\mathrm{~S}, \mathrm{D}, \mathrm{B}, \mathrm{I}$ & autochthonous \\
\hline Apareiodon piracicabae (Eigenmann, 1907) & NUP9934 & 12 & $\mathrm{~S}, \mathrm{D}$ & autochthonous \\
\hline Parodon nasus Kner, 1859 & CPUEMS138 & 12.7 & $\mathrm{~S}, \mathrm{D}, \mathrm{V}, \mathrm{B}, \mathrm{I}$ & autochthonous* \\
\hline \multicolumn{5}{|l|}{ Prochilodontidae } \\
\hline Prochilodus lineatus (Valenciennes, 1836) & CPUEMS595 & 59.2 & $\mathrm{~S}, \mathrm{D}, \mathrm{V}, \mathrm{B}, \mathrm{I}$ & autochthonous* \\
\hline \multicolumn{5}{|l|}{ Serrasalmidae } \\
\hline Metynnis lippincottianus (Cope, 1870) & NUP9380 & 14.9 & I & unknown \\
\hline Myloplus tiete (Eigenmann \& Norris, 1900)* & NUP9364 & 16.3 & I & autochthonous \\
\hline Serrasalmus maculatus Kner, 1858 & CPUEMS521 & 20.2 & $\mathrm{~S}, \mathrm{D}, \mathrm{V}, \mathrm{B}, \mathrm{I}$ & autochthonous* \\
\hline Serrasalmus marginatus Valenciennes, 1837 & CPUEMS708 & 27 & S,D,B,I & autochthonous \\
\hline
\end{tabular}




\begin{tabular}{|c|c|c|c|c|}
\hline Species & Voucher or Reference & Size (cm) & sub-basin & origin \\
\hline \multicolumn{5}{|l|}{ GYMNOTIFORMES } \\
\hline \multicolumn{5}{|l|}{ Apteronotidae } \\
\hline Apteronotus albifrons (Linnaeus, 1766) & NUP9386 & $50^{*}$ & $\mathrm{D}, \mathrm{I}$ & allochthonous \\
\hline \multicolumn{5}{|l|}{ Gymnotidae } \\
\hline Gymnotus inaequilabiatus (Valenciennes, 1839) & CPUEMS697 & $60^{*}$ & $\mathrm{D}, \mathrm{V}, \mathrm{I}$ & allochthonous \\
\hline Gymnotus omarorum Richer-de-Forges, Crampton \& Albert, 2009 & MUZEL14328 & $25.4^{*}$ & I & unknown \\
\hline $\begin{array}{l}\text { Gymnotus pantanal Fernandes, Albert, Daniel-Silva, Lopes, } \\
\text { Crampton \& Almeida-Toledo, } 2005\end{array}$ & MUZEL14486 & $25.1^{*}$ & I & autochthonous* \\
\hline Gymnotus paraguensis Albert \& Crampton, 2003 & CPUEMS179 & $24 *$ & $\mathrm{D}, \mathrm{I}$ & allochthonous \\
\hline Gymnotus sylvius Albert \& Fernandes-Matioli, 1999 & NUP9389 & $36^{*}$ & $\mathrm{~S}, \mathrm{D}, \mathrm{V}, \mathrm{B}, \mathrm{I}$ & autochthonous* \\
\hline \multicolumn{5}{|l|}{ Hypopomidae } \\
\hline Brachyhypopomus gauderio Giora \& Malabarba, 2009* & NUP9390 & $18.6^{*}$ & $\mathrm{~B}, \mathrm{I}$ & allochthonous \\
\hline \multicolumn{5}{|l|}{ Rhamphichthyidae } \\
\hline Gymnorhamphichthys britskii Carvalho, Ramos \& Albert, 2011* & NUP9384 & 11.5 & I & autochthonous* \\
\hline \multicolumn{5}{|l|}{ Sternopygidae } \\
\hline Eigenmannia trilineata López \& Castello, 1966 & NUP9381 & $25^{*}$ & $\mathrm{~S}, \mathrm{D}, \mathrm{B}, \mathrm{I}$ & autochthonous* \\
\hline Eigenmannia virescens (Valenciennes, 1847) & CPUEMS284 & $35.8^{*}$ & $\mathrm{~S}, \mathrm{~B}, \mathrm{I}$ & autochthonous* \\
\hline Sternopygus macrurus (Bloch \& Schneider, 1801) & NUP9388 & $141^{*}$ & $\mathrm{~S}, \mathrm{D}, \mathrm{B}, \mathrm{I}$ & autochthonous* \\
\hline \multicolumn{5}{|l|}{ SILURIFORMES } \\
\hline \multicolumn{5}{|l|}{ Aspredinidae } \\
\hline Bunocephalus larai Ihering, 1930 & NUP9352 & 5.6 & S,B,I & autochthonous \\
\hline \multicolumn{5}{|l|}{ Auchenipteridae } \\
\hline Auchenipterus osteomystax (Miranda Ribeiro, 1918) & CPUEMS709 & 27 & $\mathrm{~B}, \mathrm{I}$ & autochthonous* \\
\hline Tatia neivai (Ihering, 1930) & NUP9313 & 8.2 & $\mathrm{D}, \mathrm{B}, \mathrm{I}$ & autochthonous \\
\hline Trachelyopterus coriaceus Valenciennes, 1840 & MZUEL14503 & $18^{*}$ & I & unknown \\
\hline Trachelyopterus galeatus (Linnaeus, 1766) & NUP9377 & 22 & $\mathrm{D}, \mathrm{V}, \mathrm{B}, \mathrm{I}$ & autochthonous* \\
\hline \multicolumn{5}{|l|}{ Callichthyidae } \\
\hline Callichthys callichthys (Linnaeus, 1758) & NUP9359 & 17 & V,B,I & autochthonous* \\
\hline Corydoras aeneus (Gill, 1858) & NUP9362 & 7.5 & $\mathrm{~S}, \mathrm{D}, \mathrm{V}, \mathrm{B}, \mathrm{I}$ & autochthonous* \\
\hline Hoplosternum littorale (Hancock, 1828) & NUP9368 & 15.8 & B.I & autochthonous* \\
\hline Lepthoplosternum pectorale (Boulenger, 1895) & MUZEL14322 & 6 & I & unknown \\
\hline \multicolumn{5}{|l|}{ Cetopsidae } \\
\hline Cetopsis gobioides Kner, 1858 & NUP9367 & 10.9 & $\mathrm{~B}, \mathrm{I}$ & autochthonous* \\
\hline \multicolumn{5}{|l|}{ Clariidae } \\
\hline Clarias gariepinus (Burchell, 1822) & CPUEMS594 & 32.9 & $\mathrm{D}, \mathrm{V}$ & exotic \\
\hline \multicolumn{5}{|l|}{ Doradidae } \\
\hline Ossancora eigenmanni (Boulenger, 1895) & CPUEMS704 & 15.2 & $\mathrm{~S}, \mathrm{I}$ & allochthonous \\
\hline Platydoras armatulus (Valenciennes, 1840) & Agostinho et al. 1997 & 43 & B & unknown \\
\hline Pterodoras granulosus (Valenciennes, 1821) & CPUEMS273 & 60 & $\mathrm{~B}, \mathrm{I}$ & allochthonous \\
\hline Trachydoras paraguayensis (Eigenmann \& Ward, 1907) & CPUEMS224 & 19.2 & $\mathrm{D}, \mathrm{B}, \mathrm{I}$ & allochthonous \\
\hline \multicolumn{5}{|l|}{ Heptapteridae } \\
\hline Cetopsorhamdia iheringi Schubart \& Gomes, 1959 & NUP9348 & 11.1 & $\mathrm{D}, \mathrm{V}, \mathrm{I}$ & autochthonous \\
\hline Imparfinis borodini Mees \& Cala, 1989 & CPUEMS701 & 15.7 & B & autochthonous \\
\hline Imparfinis mirini Haseman, 1911 & NUP9355 & 8.5 & $\mathrm{D}, \mathrm{B}, \mathrm{I}$ & autochthonous \\
\hline Imparfinis schubarti (Gomes, 1956) & NUP9348 & 10.6 & $\mathrm{D}, \mathrm{V}, \mathrm{I}$ & autochthonous \\
\hline
\end{tabular}


Continuation Table 1.

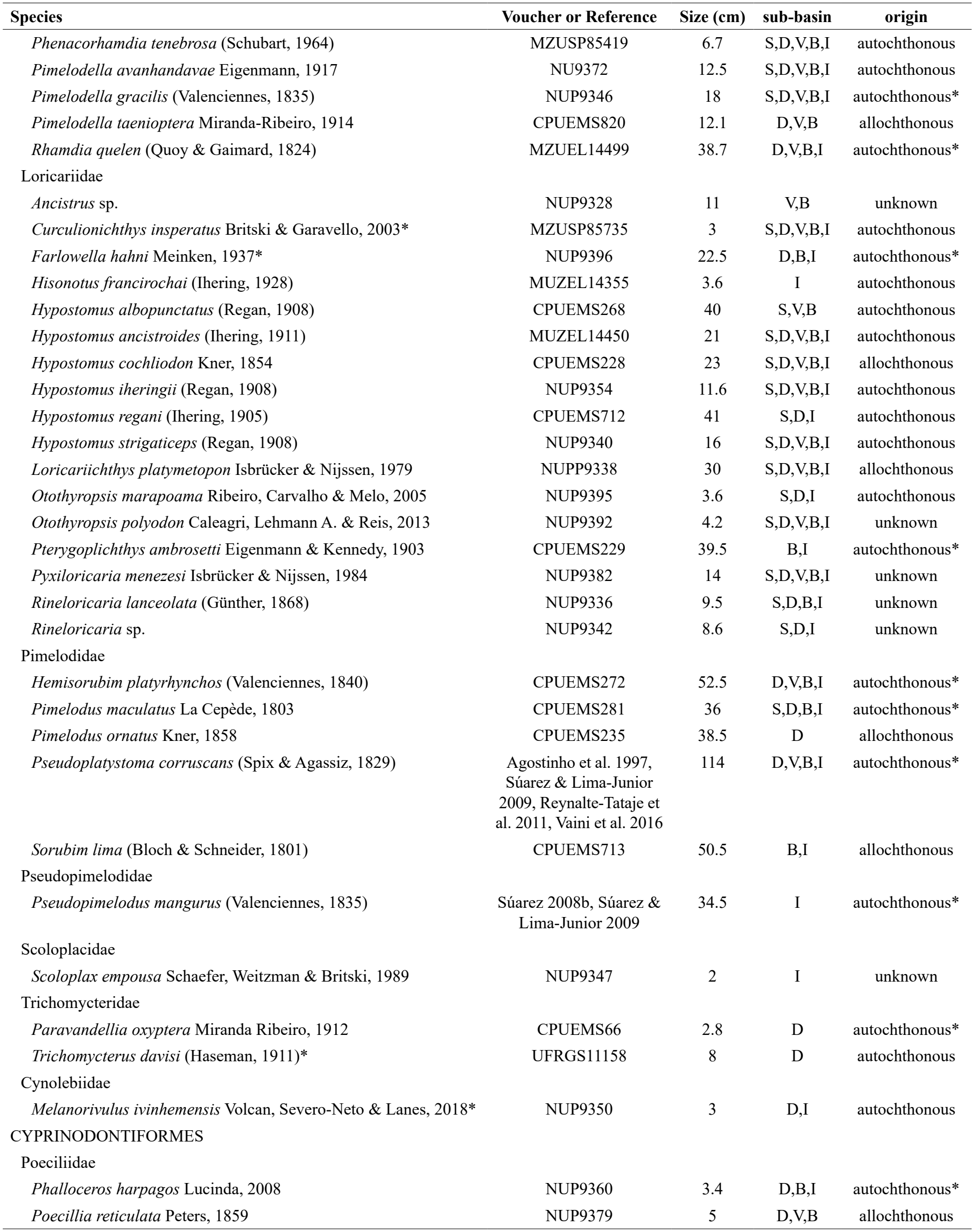


Continuation Table 1.

\begin{tabular}{|c|c|c|c|c|}
\hline Species & Voucher or Reference & Size (cm) & sub-basin & origin \\
\hline \multicolumn{5}{|l|}{ SYNBRANCHIFORMES } \\
\hline \multicolumn{5}{|l|}{ Synbranchidae } \\
\hline \multicolumn{5}{|l|}{ CICHLIFORMES } \\
\hline \multicolumn{5}{|l|}{ Cichlidae } \\
\hline Astronotus crassipinnis Heckel, 1840 & CPUEMS541 & 24 & $\mathrm{~V}, \mathrm{~B}$ & allochthonous \\
\hline Chaetobranchopsis australis Eigenmann \& Ward, 1907 & NUP9366 & 12 & I & allochthonous \\
\hline Cichlasoma dimerus (Heckel, 1840) & MZUEL14512 & 11.7 & I & unknown \\
\hline Cichlasoma paranaense Kullander, 1983 & NUP9385 & 7.8 & $\mathrm{~S}, \mathrm{D}, \mathrm{V}, \mathrm{B}, \mathrm{I}$ & autochthonous \\
\hline Coptodon rendalli (Boulenger, 1897)* & NUP9316 & 16.6 & $\mathrm{D}, \mathrm{B}, \mathrm{I}$ & exotic \\
\hline
\end{tabular}

${ }^{1}$ Species name with asterisk indicates that the deposit was made with the synonym species and it changed according to the new identification.

${ }^{2}$ Acronym refers to the following collections: Universidade Estadual de Maringá (NUP), Coleção de Peixes - Universidade Estadual Paulista/Campus de São José do Rio Preto (DZSJRP), Museu de Zoologia da Universidade de São Paulo (MZUSP), Museu de Ciências e Tecnologia da PUCRS (MCP), Universidade Federal do Rio Grande do Sul (UFRGS) and Museu de Zoologia da Universidade Estadual de Londrina (MZUEL), Coleção de Peixes da Universidade estadual de Mato Grosso do Sul (CPUEMS).

${ }^{3}$ Autochthonous: species from upper Paraná, asteristik indicates the type locality outside upper Paraná; Allochthonous: species from other Neotropical basins; Exotic: species from other continents and Unknown species no information about on the origin.

${ }^{4}$ Voucher number indicates species deposited by this work; References were used when it was not possible to deposit voucher specimens, but the species was already reported in the Ivinhema river basin; hastag, the only species without deposited specimens in the Ivinhema river basin, but reported by Langeani et al. (2007) in the upper Paraná river basin.

SÚAREZ, Y.R. \& LIMA-JÚNIOR, S.E. 2009 Spatial and temporal variation in stream fsh assemblages of Guiraí River Basin, Upper Paraná Basin. Biota Neotrop. 9(1):101-111 http://www.biotaneotropica.org.br/v9n1/en/ abstract?article+bn01709012009 (ultimo acesso em 21/01/2019)

VAINI, J.O., CRISPIM, B.A., SILVA, D.B.S., BENITES, C., RUSSO, M. R. \& GRISOLIA, A. B. 2016. Genetic variability of pure Pseudoplatystoma corruscans and Pseudoplatystoma reticulatum individuals in the Paraná and Paraguay River basins. Fish. Sci. 82:605-611.

Moenkhausia forestii, Brachyhypopomus gauderio, Laetacara araguaiae, Gymnotus omarorum, Gymnorhamphichthys britskii, Otothyropsis polyodon, Melanorivulus ivinhemensis, Astyanax abramis*, Moenkhausia oligolepis*, Serrapinnus kriegi*, Curimatopsis myersi*, Pyxiloricaria menezesi ${ }^{*}$, Cichlasoma dimerus ${ }^{*}$, Characidium lagosantense, Rineloricaria lanceolata, Moenkhausia bonita, Metynnis lippincottianus, Farlowella hahni, Trichomycterus davisi, Apistogramma commbrae, Chaetobranchopsis australis, Psellogrammus kennedyi, and Serrapinnus calliurus. Some species may be visualized in Figure 2.

Regarding to geographic origin, 93 species were autochthonous from the Paraná river (42 from upper Paraná and 51 from lower Paraná), 27 were allochthonous and two were exotics (Table 1). It was not possible to define the origin of 19 species, mainly because nothing is reported about them or because the species are yet to be described.

The length of fish species varied from two to $150 \mathrm{~cm}$. Small-sized fish were mostly common $(63.8 \%, 90$ species $)$ in size class until 20.7 $\mathrm{cm}$ (Figure 3), fish of medium size were in the second size class $39.4 \mathrm{~cm}$ $(19.9 \%, 28$ species $)$ and the other 16 species $(16.3 \%)$ were considered large size, above $39.5 \mathrm{~cm}$ and until $150 \mathrm{~cm}$.

\section{Discussion}

For the upper Paraná river basin it was reported 317 described fish species by Langeani et al. (2007). Although, since it was published this list may be increased, in function of the new descriptions, our study has 117 identified species (35.6\%) in common, it can be said that the Ivinhema river basin, covering only $5 \%$ of the total area of the upper Paraná, possesses an expressive fish fauna, one third of the species.

From 24 species not reported on the inventory of Langeani et al. (2007), eight were described after the publication: P. harpagos, M. forestii, B. gauderio, L. araguaiae, G. omarorum, G. britskii, $O$. polyodon, and Melanorivulus ivinhemensis (Lucinda 2008, Benine et al. 2009, Giora \& Malabarba 2009, Ottoni \& Costa 2009, Richerde-Forges et al. 2009, Carvalho et al. 2011, and Calegari et al. 2013, Volcan et al. 2018, respectively). Phallocerus harpagos, $M$. forestii, $G$. britskii, O. polyodon, and M. ivinhemensis were already described with specimens sampled in the upper Paraná river basin. It is noteworthy that M. ivinhemensis was described recently and is considered endemic in marginal areas of small first order streams and wetlands in the Ivinhema river (Ota et al. 2018). Brachyhypopomus gauderio was described from 


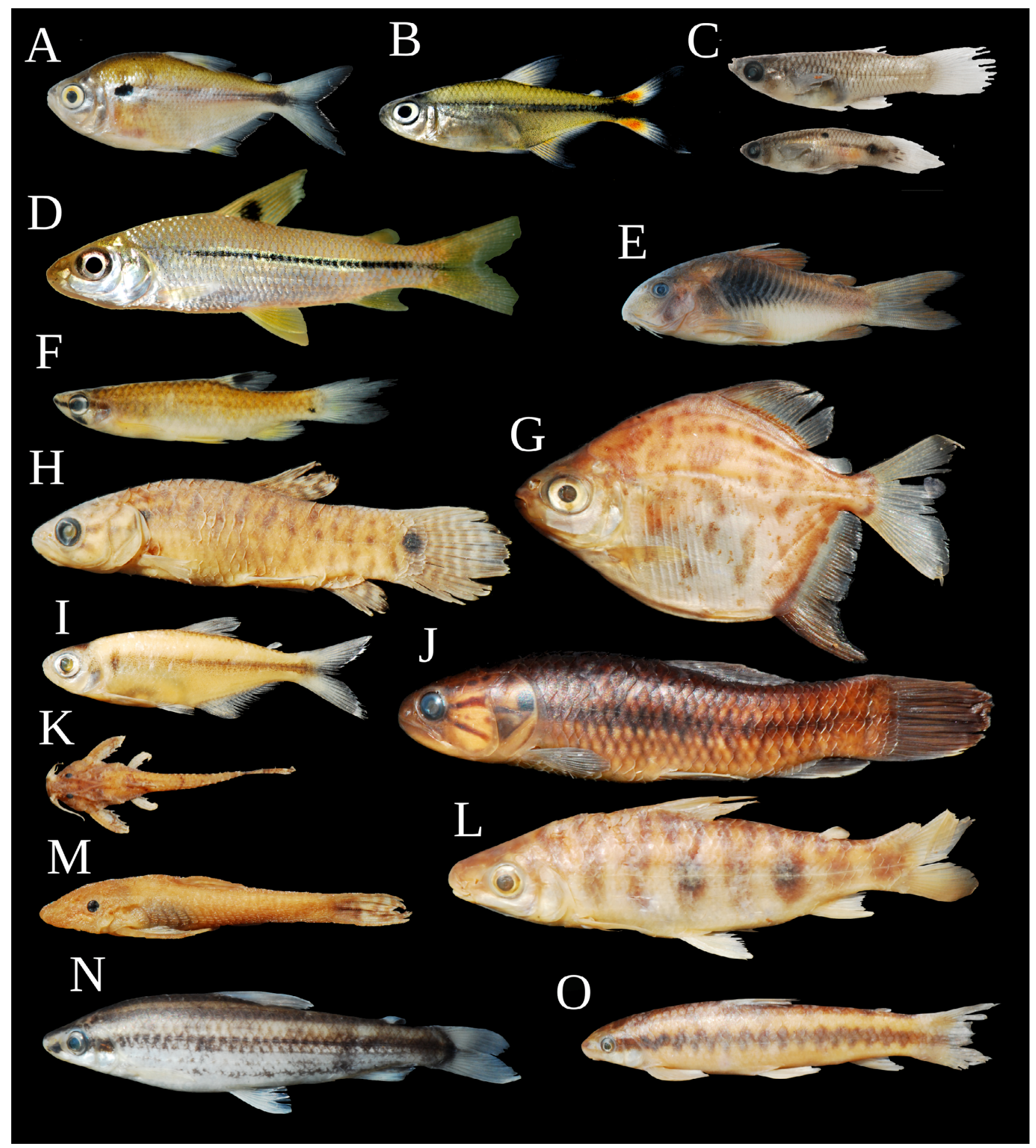

Figure 2. Representative specimens for some fish species reported in the Ivinhema river basin, in the upper Paraná river basin, Mato Grosso do Sul State, Brazil. A) Astyanas lacustris; B) Hemigrammus marginatus; C) (Female and male) Poecilia reticulata; D) Steindachnerina brevipinna; E) Corydoras aeneus; F) Pyrrhulina australis; G) Myloplus tiete; H) Erythrinus erythrinus; I) Knodus moenkhausii; J) Hoplerythrinus unitaeniatus; K) Scoloplax empousa; L) Leporinus octofasciatus; M) Otothyropsis polyodon; N) Leporinus striatus; O) Paradon nasus. 


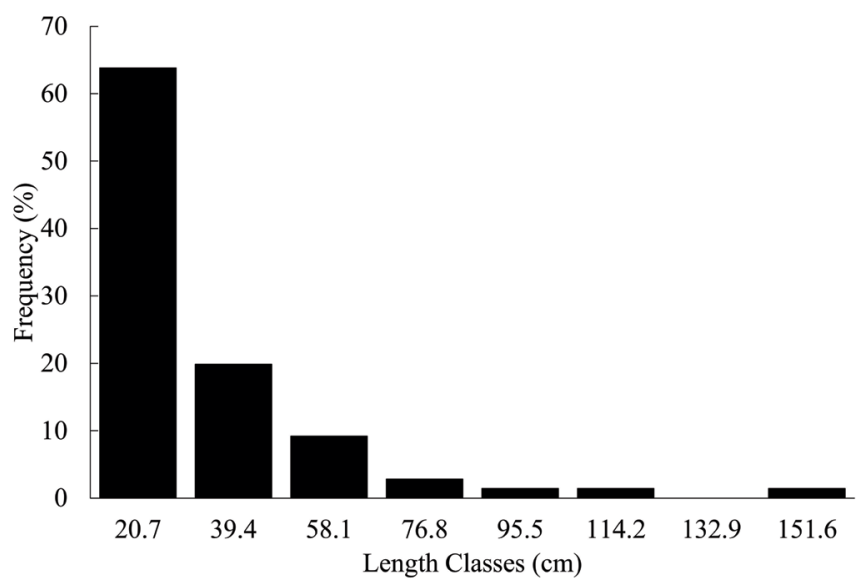

Figure 3. Frequency of fish by size classes along the Ivinhema river basin, upper Paraná river basin, Mato Grosso do Sul State, Brazil.

Paraguay and Uruguay basins and Brazilian southeastern cost (Giora \& Malabarba 2009), but according to Ota et al. (2018) this species was identified as B. cf. pinnicaudatus (Graça \& Pavanelli 2007) and its occurrence in the upper Paraná might be associated to introduction as a live bait by anglers. Laetacara aragauaiae was described from the Verde and Araguaia rivers (Ottoni \& Costa 2009), but as a non-native species, it was reported the upper Paraná floodplain, probably introduced by the aquarium trade (Ota et al. 2018), and now we expanded its occurrence to the river Ivinhema.

For six species, the current study had extended their occurrence to the upper Paraná river: Astyanax bimaculatus subgroup was revised by Lucena \& Soares (2016) and they re-described $A$. abramis as a valid species from Paraguay, Lower Paraná and La Plata river basin. Moenkhausia oligolepis was reported originally only in the Guianas and Amazon river basins, but Benine et al (2009) with a detailed revision of this species reported its occurrence in Paraguay river. For S. kriegi, C. myersi, P. menezesi and C. dimerus and the other above-mentioned species, there was not found any report for these six species in the upper Paraná, thus these are the first reports.

Ten species not reported in Langeani et al. (2007) had their taxonomic status revised or occurrence extended. Characidium lagosantense was re-described and reported in the Mogi-Guaçu river by Silveira (2008). The taxonomic revision of Rineloricaria species was carried out in the Paraguay river (Vera-Alcaráz et al. 2012), but $R$. lanceolata occurrence was also recorded in the Ivinhema river in 2011 and reported by Froehlich et al. (2017). Moenkhausia bonita was described in 2004 in the Paraguay river (Benine et al. 2004), but its occurrence in the Ivinhema river was registered in 2006 and reported by Froehlich et al. (2017) and Ota et al. (2018). Metynnis lippincottianus was reported in the Ivinhema river by Súarez (2011) and Froehlich et al. (2017). It is interesting to point out that M. lippincottianus was not reported by Langeani et al. (2007) and it was adverted that it had been identified in the upper Paraná as M. maculatus (Graça \& Pavanelli 2007). However, as an original Amazonian fish, two populations of this species were reported in two tributary streams of Ilha Solteira dam, suggesting these populations were installed locally (Froehlich et al. 2017) and now our study reported this species in many streams in the Ivinhema river. Its occurrence can be associated with the releasing of specimens for restocking or with the aquarium trade (Ota 2015).
Farlowella hahni, despite not being reported by Langeani et al. (2007), it was reported in the upper Paraná by Graça \& Pavanelli (2007) as F. amazonum, but Ota et al. (2018) comparing the characters, described for the genus by Retzer \& Page (1996), decided that the species would match more with the description of $F$. hahni. This species was reported before in the lower Paraná river and its occurrence in the upper portion can be associated to the inundation of the Sete Quedas Falls after the filling of Itaipu Reservoir (Ota et al. 2018). Trichomycterus davisi, first identified as Trichomycterus sp. (Graça \& Pavanelli 2007), has now been assigned as $T$. davisi belonging to a species complex (Ota et al. 2018). Apistogramma commbrae and C. australis may have been introduced accidentally by aquarists or even after the construction of the Itaipu dam, since these species originally occurred only in the lower Paraná river and were firstly reported in the upper Paraná (Ivinhema river) by Súarez et al. (2011). Psellogrammus kennedyi, also reported for this latest author, and $S$. calliurus are considered a not-native species in the upper Paraná and had their occurrence associated with the function of a fish ladder that connects downstream portion (lower Paraná) to upstream portion (upper Paraná) in the Itaipu Dam (Ota et al. 2018).

Whereas Langeani et al. (2007) estimated at least 50 more species to be described, our inventory shows that the true richness of the Ivinhema river basin may be even greater insofar as specific identification was not possible for four species (four species at genus level). According to taxonomists, we probably have four new species: Serrapinnus sp., Ancistrus sp., and Rineloricaria sp. (personal communication). Thus, even without considering other species with uncertain taxonomic definitions, $2.8 \%$ of the sampled species are poorly or completely unknown to science.

The greater representation of the orders Characiformes and Siluriformes is a frequent pattern in Neotropical waters (Castro et al. 2004). Both orders accounted for $81.6 \%$ of the sampled species, which is consistent with that found by Langeani et al. (2007). This pattern is not surprising since both orders are the most representative of South America freshwater fish (Lowe-Mcconnell 1999). On the other hand, the greater representation of the families Characidae and Loricariidae reflects a recurrent pattern in Brazilian continental waters (Reis et al. 2003, Buckup et al. 2007).

Although the majority of fish reported in the Ivinhema river basin was autochthonous $(29.8 \%)$ in the upper Paraná and autochthonous in the lower Paraná $(36.2 \%)$, summing $66 \%$, there was a substantial occurrence of allochthonous species (20\%). The two exotic species recorded (1.4\%), Clarias gariepinus and Coptodon rendalli, were clearly introduced into the basin through pisciculture (Langeani et al. 2007). This is the first report of C. gariepinus in the Dourados and Vacaria rivers, expanding the knowledge about their occurrence in tributaries of upper Paraná, which is considered to be highly adaptable and capable of negatively affecting the native fish fauna (Alves et al. 1999). During sampling, local fishermen and riverine communities reported that this species is frequently caught in these sub-basins and in abundance in the Dourados river.

Even though we do not have knowledge about the origin of $13.5 \%$ of the species, a point that is very concerning in this inventory is that only $29.8 \%$ of species may be considered native from the upper Paraná, the remaining fishes $(56.7 \%)$ are from the lower Paraná, other basins or other continents. When the subject is homogenization and species 
invasion, it is important to consider not only allochthonous and exotic species. Introduced species from different parts of the basin may be more problematic than species from other basins or continents (Vitule 2009). In Paraná river, even the species that overcame the barrier from the lower to the upper parts, because of the impoundment caused by Itaipu Dam, may cause some troubles for upper communities, such as pointed by Taylor et al. (1984): hybridization, genetic losses, trophic alterations, parasites or diseases introduction. Those alterations in local communities may result in extinction of native species, biodiversity losses or biota homogenization (Vitule 2009).

The streams of the present study exhibited greater species richness (112) than streams of other works in upper Paraná, such as Castro et al. (2003, 2004), Gubiani et al. (2006) e Gomiero \& Braga (2006), which reported 52, 64, 62 and 48 species, respectively. In spite of these researches have used different methodologies and our samples were carried out in more stretches and in a greater area, consequently reflecting on difference catch effort and species richness, the rivers of the Paraná river basin in the state of São Paulo have experienced the influence of a series of impoundments for hydroelectric reservoirs, a process that affects sub-basin connections, thereby preventing recolonization and, thus, influencing species richness. On the other hand, the Ivinhema river basin, in the western portion of the upper Paraná, does not contain significant impoundments, with only two small hydropower plants in the upper portion of the Dourados river (São João I and II), or any other barriers, thus maintaining the potential for greater connectivity among streams, facilitating species flux and even recolonization by species from the Paraná river floodplain.

The most common species sampled were Astyanax lacustris, Serrapinnus notomelas, Piabarchus stramineus, and Hypostomus ancistroides. These small-sized species have great feeding plasticity and are known for their capacity to adapt to and colonize different water bodies. These species were reported throughout the Ivinhema river basin and are widely distributed in the upper Paraná river (Castro et al. 2004, Gubiani et al. 2006, Teresa \& Casatti 2010, Viana et al. 2013).

A wide range of standard length was registered for the sampled fish ( 2 to $150 \mathrm{~cm}$ ), however, it was evidenced that the fish fauna of Ivinhema river Basin is composed basically of small-sized species, about $63.8 \%$ smaller than $20.7 \mathrm{~cm}$. Despite of a greater number of samples in streams, and this may influence in a higher richness of small-sized fish (Castro 1999, Castro et al. 2003, 2004, Couto \& Aquino 2011), this may be considered a pattern for the basin, because the large-sized species were sampled mainly below the intermediate altitude $(430 \mathrm{~m})$ and in larger rivers, in areas located in tributaries such as Dourados, Brilhante and Ivinhema rivers. Moreover, $65 \%$ of 310 species analyzed in the upper Paraná river were small-sized $(\leq 20.8 \mathrm{~cm}$ ) (Langeagni et al., 2007) and $50 \%$ of South-American freshwater fish are small-sized (Castro 1999). According to this last work, this pattern may be a result of the high hydrological variability in streams; we believe that this pattern act more intensively structuring fish assemblages locally, where the water levels may vary between rainy and dry periods until five meters (personal observation), selecting generalist and resilient small-sized fish that are more prone to establish in these areas.

In conclusion, the current study is particularly significant because it helps reduce the lack of information on fish communities of an ecosystem that has been kept relatively free from dams and impoundments, from its headwaters to its confluence with the Paraná river basin. These data are also important for understanding the distribution of fish among sub-basins, adding new species records for the Ivinhema river basin, and the upper Paraná basin as a whole, serving as reference data for each region and contributing to the management, decision-making, and implementation of politics to conserve the basin as a whole. Furthermore, these data should prove useful as a reference for monitoring the fish fauna of the basin, which has experienced great alterations to its landscapes over the last decade due to the expansion of sugarcane crops and also may be suffering some pressure by a high percentage of non-native species in the upper Paraná.

\section{Acknowledgments}

The authors acknowledge the financial support of Fundação de Apoio ao Desenvolvimento do Ensino, Ciência e Tecnologia do Estado de Mato Grosso do Sul (FUNDECT), Conselho Nacional de Desenvolvimento Científico e Tecnológico (CNPq), and of Centro de Pesquisas do Pantanal (CPP/MCT). W. Vicentin and F. S. Ferreira are grateful for the doctorate fellowship and logistic support provided by the Coordenação de Aperfeiçoamento de Pessoal de Nível Superior (CAPES). Y. R. Súarez is supported by productivity grants from Conselho Nacional de Desenvolvimento Científico e Tecnológico (CNPq). The authors are also grateful to logistic support in field and in the laboratory to João P. da Silva, Karina K. Tondato, Alexandro C. Florentino, Luzia S. Lourenço, Patrícia L. Rondon, Aryadne S. Rocha, Maiane J. Pereira, Viviane V. Azevedo, Gabriela S. V. Duarte, Marcelo M. de Souza, Mariane I. Santos, Lidiani Q. L. Ximenes, Paula S. Doreto, and Ana P. Lemke. For the help in identifying fish species, acknowledgments are also due to: Dra. Carla S. Pavanelli and Dr. Cláudio Henrique Zawadzki at Universidade Estadual de Maringá (NUP), Dr. Flavio Lima at Museu de Zoologia da Universidade de São Paulo (MZUSP), currently at Universidade Estadual de Campinas, Dr. Fernando C. Jerep at Museu de Zoologia da Universidade Estadual de Londrina (MZUEL).

\section{Author Contributions}

Wagner Vicentin: Substantial contribution in the concept and design of the study; contribution to data collection; Contribution to data analysis and interpretation; Contribution to manuscript preparation; Contribution to critical revision, adding intellectual content.

Fabiane Silva Ferreira: Substantial contribution in the concept and design of the study; contribution to data collection; Contribution to data analysis and interpretation; Contribution to manuscript preparation; Contribution to critical revision, adding intellectual content.

Yzel Rondon Súarez:Substantial contribution in the concept and design of the study; contribution to data collection; Contribution to data analysis and interpretation; Contribution to manuscript preparation; Contribution to critical revision, adding intellectual content.

\section{Conflicts of interest}

The authors declare that they have no conflict of interest related to the publication of this manuscript. 


\section{References}

AGOSTINHO, A.A., GOMES, L.C. \& PELICICE, F.M. 2007. Ecologia e manejo de recursos pesqueiros em reservatórios do Brasil. EDUEM, Maringá.

AGOSTINHO, A.A., GOMES, L.C., SUZUKI, H.I. \& JÚLIO JUNIOR, H.F. 2003. Migratory fishes of the Upper Paraná River Basin Brazil. In Migratory Fishes of South America: Biology, Fisheries and Conservation Status (J. Carolsfeld, B. Harvey, C. Ross \& A. Baer, eds.). World Bank, Vitoria, p.19-89.

AGOSTINHO, A.A., JÚLIO-JÚNIOR, H.F., GOMES, L.C., BINI, L.M. \& AGOSTINHO, C.S. 1997. Composição, abundância e distribuição espaçotemporal da ictiofauna. In A planície de inundação do ato rio Paraná: aspectos físicos, biológicos e socioeconômicos (A.E.A.M. Vazzoler, A.A. Agostinho \& N.S. Hahn, eds.). EDUEM, Maringá, p.179-208.

AGOSTINHO, A.A., THOMAZ, S.M., MINTE-VERA, C.V. \& WINEMILLER, K.O. 2000. Biodiversity in the high Paraná riverfloodplain. In Biodiversity in wetlands: assessment, function and conservation. (B. Gopal, W.J. Junk \& J.A. Davis, eds.). Backhuys Publishers, Leiden, p. 89-118.

ALVES, C.B.M., VONO, V. \& VIEIRA, F. 1999. Presence of the walking catfish Clarias gariepinus (Burchell) (Siluriformes, Clariidae) in Minas Gerais state hydrographic basins, Brazil. Rev. Bras. Zool. 16(1):259-263.

AQUINO, P.P.U., SCHNEIDER, M., MARTINS-SILVA, M.J., PADOVESIFONSECA, C., ARAKAWA, H.B. \& CAVALCANTI, D.R. 2009. Ictiofauna dos córregos do Parque Nacional de Brasília, bacia do Alto Rio Paraná, Distrito Federal, Brasil Central. Biota Neotrop. 9(1): 217-230 http://www. biotaneotropica.org.br/v9n1/pt/abstract?inventory+bn02809012009 (last access in 20/02/2014).

BENINE, R.C., CASTRO, R.M.C. \& SABINO, J. 2004. Moenkhausia bonita: A new small characin fish from the Rio Paraguay basin, southwestern Brazil (Characiformes: Characidae). Copeia. 4(1):68-73.

BENINE, R.C., MARIGUELA, T.C. \& OLIVEIRA, C. 2009. New species of Moenkhausia Eigenmann, 1903 (Characiformes: Characidae) with comments on the Moenkhausia oligolepis species complex. Neotrop. Ichthyol. 7(2):161-168.

BRYCE, S.A., LARSEN, D.P., HUGHES, R.M. \& KAUFMANN, P.R. 1999. Assessing relative risks to aquatic ecosystems: a mid-Appalachian case study. J. Am. Water Resour. As. 35(1):23-36.

BUCKUP, P.A., MENEZES, N.A. \& GHAZZI, M.S. 2007. Catálogo das espécies de peixes de água doce do Brasil. Museu Nacional, Rio de Janeiro.

BULLA, C.K., GOMES, L.C., MIRANDA, L.E. \& AGOSTINHO, A.A. 2011. The ichthyofauna of drifiting macrophyte mats in the Ivinheima River, Upper Paraná River Basin, Brazil. Neotrop. Ichthyol. 9(2):403- 409.

CALEGARI, B.B., LEHMANN, P.A. \& REIS. R.E. 2013. Two new species of cascudinhos of the genus Otothyropsis (Siluriformes: Hypoptopomatinae) from the rio Paraná basin, Brazil. Zootaxa. 3619(2):130-144.

CARVALHO, T.P., RAMOS C.S. \& ALBERT J.S. 2011. A new species of Gymnorhamphichthys (Gymnotiformes: Rhamphichthyidae) from the Paraná-Paraguay basin. Copeia, 2011(3): 400-406

CASTRO, R.M.C. 1999. Evolução da ictiofauna de riachos sul-americanos: padrões gerais e possíveis processos causais. In Ecologia de peixes de riachos (E.P. Caramaschi, R. Mazzoni \& P.R. Peres-Neto, eds.). PPGE-UFRJ, Rio de Janeiro, p.139-155.

CASTRO, R.M.C., CASATTI, L., SANTOS, H.F., FERREIRA, K.M., RIBEIRO, A.C., BENINE, R.C., DARDIS, G.Z.P., MELO, A.L.A., STOPLIGLIA, R., ABREU, T.X., BOCKMANN, F.A., CARVALHO, M., GIBRAN, F.Z. \& LIMA, F.C.T. 2003. Estrutura e composição da ictiofauna de riachos do Rio Paranapanema, Sudeste e Sul do Brasil. Biota Neotrop. 3(1): 1-14 http:// www.biotaneotropica.org.br/v3n1/pt/abstract?article+BN01703012003 (last access in 20/12/2013).

CASTRO, R.M.C., CASATTI, L., SANTOS, H.F., MELO, A.L.A., MARTINS, L.S.F., FERREIRA, K.M., GIBRAN, F.Z., BENINE, R.C., CARVALHO, M., RIBEIRO, A.C., ABREU, T.X., BOCKMANN, F.A., PELIÇÃO, G.Z., STOPLIGLIA, R. \& LANGEANI, F. 2004. Estrutura e composição da ictiofauna de riachos da bacia do Rio Grande no estado de São Paulo, sudeste do Brasil. Biota Neotrop. 4(1): 1-39 http://www.biotaneotropica.cria.org. br/v4n1/pt/download?article+BN01704012004 (last access in 20/12/2013).
COUTO, T.B.A. \& AQUINO, P.D.P.U. 2011. Structure and integrity of fish assemblages in streams associated to conservation units in Central Brazil. Neotrop. Ichthyol. 9(2):445-454.

DOMINGUES, T.A. \& THOMAZ JUNIOR, A. 2012. A territorialização da canade-açúcar no Mato Grosso do Sul. Cad. Prudentino Geogr. 34(1):138-160.

ESCHMEYER, W.N., FRICKE, R. \& VAN DER LAAN, R. (Eds). 2018. Catalog of Fishes: Genera, Species, Reference http://researcharchive.calacademy. org/research/ichthyology/catalog/fishcatmain.asp. Electronic version (las access in 31/05/2018)

FISHBASE. http://www.fishbase.org/home.htm (last access in 15/12/2017)

FROEHLICH, O., CARVALHO, M., SABINO, J., SÚAREZ, Y.R. \& VILELA, M.J.A. 2017. Checklist da ictiofauna do Estado de Mato Grosso do Sul, Brasil. Iheringia, Sér. Zool. 107(supl.):e2017151.

FROESE, R. \& PAULY, D. (Eds). 2018. FishBase. World Wide Web electronic publication. http://www.fishbase.org, version (02/2018). (last access in 31/05/2018)

GIORA, J. \& MALABARBA, L.R. 2009, Brachyhypopomus gauderio, new species, a new example of underestimated species diversity of electric fishes in the southern South America (Gymnotiforme, Hypopomidae). Zootaxa. 2093:60-68.

GOMIERO, L.M. \& BRAGA, F.M.S. 2006. Ichthyofauna diversity in a protected area in the state of São Paulo, southeastern Brazil. Braz. J. Biol. 66(1):75-83.

GRAÇA, W.J. \& PAVANELLI, C.S. 2007. Peixes da planície de inundação do Alto Rio Paraná e áreas adjacentes. EDUEM, Maringá.

GUBIANI, E.A., HOLZBACH, A.J., BAUMGARTNER, G., NETO, L.B.R. \& BERGMANN, F. 2006. Fish, Piquiri River, Upper Paraná River Basin, Paraná State, Brazil. Check List 2(3):9-14.

HOLT, T.V., MURPHY, D.M. \& CHAPMAN, L. 2006. Local and landscape predictors of fish-assemblage characteristics in the Great Swamp, New York. Northeast. Nat. 13(3):353-374.

LACERDA, A.C.F., TAKEMOTO, R.M. \& PAVANELLI, G. C. 2008. Digenea, Nematoda, Cestoda, and Acanthocephala, parasites in Potamotrygonidae (Chondrichthyes) from the upper Paraná River floodplain, states of Paraná and Mato Grosso do Sul, Brazil. Check List, 4(2):115-122.

LANGEANI, F., CASTRO, R.M.C., OYAKAWA, O.T., SHIBATTA, O.A., PAVANELLI, C.S. \& CASATTI, L. 2007. Diversidade da ictiofauna do Alto Rio Paraná: composição atual e perspectivas futuras. Biota Neotrop. 7(3): 181-197 http://www.biotaneotropica.org.br/v7n3/pt/abstract?article+bn03407032007 (last access in 18/02/2013)

LONARDONI, A.P., GOULART, E., OLIVEIRA, E.F. \& ABELHA, M. 2006. Hábitos alimentares e sobreposição trófica das raias Potamotrygon falkneri e Potamotrygon motoro (Chondrichthyes: Potamotrygonidae) na planície alagável do alto rio Paraná; Brasil. Acta Sci. Bio. Sci. 28(3):195-202

LOWE-MCCONNELL, R.H. 1999. Estudos ecológicos de comunidades de peixes tropicais. EDUSP, São Paulo.

LUCENA, C.A.S. \&. SOARES, H.G. 2016. Review of species of the Astyanax bimaculatus "caudal peduncle spot" subgroup sensu Garutti \& Langeani (Characiformes, Characidae) from the rio La Plata and rio São Francisco drainages and coastal systems of southern Brazil and Uruguay. Zootaxa. 4072(1):101-125

LUCINDA, P.H.F. 2008. Systematics and biogeography of the genus Phalloceros Eigenmann, 1907 (Cyprinodontiformes: Poeciliidae: Poeciliinae), with the description of twenty-one new species. Neotrop. Ichthyol. 6(2):113-158.

NASCIMENTO, F.L. \& NAKATANI, K. 2006. Relações entre fatores ambientais e a distribuição de ovos e larvas de peixes na sub-bacia do rio Ivinhema, Estado de Mato Grosso do Sul, Brasil. Acta Sci. Bio. Sci. 28(2):117-122.

OTA, R.P. 2015. Revisão taxonômica e análise filogenética das espécies do gênero Metynnis Cope, 1878 (Characiformes: Serrasalmidae). Tese de doutorado, Instituto Nacional de Pesquisas da Amazônia, Manaus, AM.

OTA, R.R., DEPRÁ, G.D., WEFERSON JÚNIO DA GRAÇA, W.J. \& PAVANELLI, C.S. 2018. Peixes da planície de inundação do alto rio Paraná e áreas adjacentes: revised, annotated and updated. Neotrop. Ichthyol. 16(2): e170094. 
OTTONI, F.P. \& COSTA, W.J.E.M. 2009. Description of a new species of Laetacara Kullander, 1986 from central Brazil and re-description of Laetacara dorsigera (Heckel, 1840) (Labroidei: Cichlidae: Cichlasomatinae). Vertebr. Zool. 59(1): 41-48.

REIS, R.E., KULLANDER, S.O. \& FERRARIS JUNIOR, C.J. 2003. Check list of the freshwater fishes of South and Central America. EDPUCRS, Porto Alegre.

RETZER, M.E. \& PAGE, L.M. 1996. Systematics of the stick catfshes, Farlowella Eigenmann \& Eigenmann (Pisces, Loricariidae). Proc. Acad. Nat. Sci. Philadelphia. 147:33-88.

REYNALTE-TATAJE, D.A., NAKATANI, K., FERNANDES, R., AGOSTINHO, A.A., BIALETZKI, A. 2011. Temporal distribution of ichthyoplankton in the Ivinhema River (Mato Grosso do Sul State/Brazil): influence of environmental variables. Neotrop. Ichthyol. 9(2):427-436.

RICHER-DE-FORGES, M.M., CRAMPTON, W.G.R. \& ALBERT, J.S. 2009 A new species of Gymnotus (Gymnotiformes, Gymnotidae) from uruguay: description of a model species in neurophysiological research. Copeia. 2009(3): 538-544.

SILVEIRA, L.G.G. 2008. Revisão taxonômica de Characidium lagosantense Travassos 1947 (Crenuchidae: Characiformes: Ostariophysi), com descrição de uma nova espécie para o Alto Rio Paraná. M.S. Dissertation, Universidade Estadual Paulista, São José do Rio Preto.

SÚAREZ, Y.R. 2008a. Spatial and temporal variation in fish species diversity and composition in streams of Ivinhema River Basin, Upper Paraná River. Biota Neotrop. 8(3):197-204 http://www.biotaneotropica.org.br/v8n3/ en/ abstract?article+bn02308032008 (last access in 22/12/2017)

SÚAREZ, Y.R. 2008b. Fish, Lower Ivinhema River Basin streams, state of Mato Grosso do Sul, Brazil. Check List 4(3): 226-231.

SÚAREZ, Y.R. \& LIMA-JÚNIOR S.E. 2009 Spatial and temporal variation in stream fish assemblages of Guiraí River basin, upper Paraná Basin. Biota Neotrop. 9(1):102-111. http://www.biotaneotropica.org.br/v9n1/en/ abstract?article+bn01709012009. (last access in 31/05/2018)

SÚAREZ, Y.R, SOUZA, M.M., FERREIRA, F.S., PEREIRA, M.J., SILVA, E.A., XIMENES, L.Q.L., AZEVEDO, L.G., MARTINS, O.C. \& LIMA JÚNIOR, S.E. 2011. Patterns of species richness and composition of fish assemblages in streams of the Ivinhema River Basin, Upper Paraná River. Acta Limnol. Bras. 23(2):177-188
SÚAREZ, Y.R., VALÉRIO, S.B., TONDATO, K.K., XIMENES, L.Q.L. \& FELIPE, T.R.A. 2007. Determinantes ambientais da ocorrência de espécies de peixes em riachos de cabeceira da bacia do rio Ivinhema, alto rio Paraná. Acta Sci. Bio. Sci. 29(2):145-150.

TAYLOR, J.N., COURTENAY, W.R.Jr., McCANN, J.A. 1984. Known impacts of exotic fishes in the continental United States. In Distribution, biology, and management of exotic fishes (W.R Courtenay Jr. \& J.R. Stauffer, eds.). Johns Hopkins University Press, Baltimore, p. 322-373.

TERESA, F.B. \& CASATTI, L. 2010. Importância da vegetação ripária em região intensamente desmatada no sudeste do Brasil: um estudo com peixes de riacho. Panam. J. Aquat. Sci. 5(3):444-453.

THOMAZ, S.M., AGOSTINHO, A.A. \& HAHN, NS. 2004. The Upper Paraná river and its Floodplain: physical aspects, ecology and conservation. Backhuys Publishers, Leiden.

VERA-ALCARAZ, H.S., PAVANELLI, C.S. \& ZAWADZKI, C.H. 2012. Taxonomic revision of the Rineloricaria species (Siluriformes: Loricariidae) from the Paraguay River basin. Neotrop. Ichthyol. 10(2):285-311.

VAINI, J.O., CRISPIM, B.A., SILVA, D.B.S., BENITES, C., RUSSO, M. R. \& GRISOLIA, A. B. 2016. Genetic variability of pure Pseudoplatystoma corruscans and Pseudoplatystoma reticulatum individuals in the Paraná and Paraguay River basins. Fish. Sci. 82:605-611.

VIANA, L.F., SÚAREZ, Y.R. \& LIMA-JÚNIOR, S.E. 2013. Influence of environmental integrity on the feeding biology of Astyanax altiparanae Garutti \& Britski 2000 in the Ivinhema river basin. Acta Sci. Bio. Sci. 35(4):541-548.

VITULE, J.R.S. 2009. Introduction of fishes in Brazilian continental ecosystems: Review, comments and suggestions for actions against the almost invisible enemy. Neotrop. Biol. Cons. 4(2):111-122.

VOLCAN, V.M., SEVERO-NETO, F. \& LANÉS, L.E.K. 2018.Unrecognized biodiversity in a world's hotspot: three new species of Melanorivulus (Cyprinodontiformes: Rivulidae) from tributaries of the right bank of the Rio Paraná basin, Brazilian Cerrado. Zoosyst. Evol. 94(2):263-280.

Received: $22 / 12 / 2017$

Accepted: 06/06/2019

Published online: 15/07/2019 